\title{
Involvement of microsomal prostaglandin E synthase-1 (mPGES-1) in diet-induced adiposity
}

\author{
Pierre Clément $^{1 \& 2}$, Airault Coraline ${ }^{1}$, Baril Nathalie ${ }^{3}$, Rim Barbouche ${ }^{1}$, Guillebaud Florent ${ }^{1}$, \\ Rami Stéphanie $^{1}$, Save Etienne ${ }^{1}$, Dallaporta Michel $^{1}$, Bariohay Bruno ${ }^{2}$ and Troadec Jean Denis ${ }^{1}$ \\ ${ }^{1}$ Aix Marseille Université. Laboratoire de Neurosciences Cognitives, UMR CNRS 7291, France, ${ }^{2}$ Biomeostasis CRO, \\ La PennelHuveaune, France and ${ }^{3}$ Fédération de Recherche 3C (FR 3512), Campus St Charles, Marseille.
}

Low grade inflammation is known to be linked to obesity, and to occur in the early stages of the disease ${ }^{(1)}$. This mechanism is complex and involves numerous organs, cells, and cytokines. In this context, inflammation of white adipose tissue seems to play a key role in the development of obesity ${ }^{(2)}$. Because of its properties, prostaglandin E2 (PGE2), an emblematic inflammatory mediator, has been proposed as an actor linking inflammation and obesity. Indeed, PGE2 is involved in mechanisms that are dysregulated in obesity such as lipolysis ${ }^{(3)}$ and adipogenesis ${ }^{(4)}$. Microsomal prostaglandin E synthase-1 (mPGES-1) is an enzyme which specifically catalyzes the final step of PGE2 biosynthesis.

Interestingly, mPGES-1 invalidation dramatically alters the production of PGE2 during inflammation ${ }^{(5)}$. In the present work, we sought to determine whether mPGES-1 could contribute to inflammation associated with obesity. First, mRNA expression of mPGES-1 was analyzed using Real-Time PCR, in epididymal white adipose tissue (EAT) of C57BL/6 mice fed with either a normal chow (NC), or a high-fat diet (HFD) during 10 weeks. In a second step, mPGES-1 - / - (KO) mice and wild-type (WT) mice, obtained from heterozygote mating, were also fed with a NC, or a HFD. Body weight and food intake were measured twice a week, and total adiposity was assessed by magnetic resonance imaging (MRI) one week before the end of the HFD period. Then, animals were sacrificed and EAT was collected for RT-PCR analysis. All results are represented as mean \pm SEM.

We observed that C57BL/6 mice fed with a HFD during 10 weeks exhibited a significant increase in body weight gain, and a decrease in mRNA expression of mPGES-1 in EAT (table 1). On the other hand, KO mice did not exhibited significant changes in body weight gain after high fat feeding, when compared with their respective control i.e. WT-HFD (table 2). Consistently, MRI acquisition showed that WT-HFD mice exhibited an important increase in total adiposity, but this increase was partially blunted in KO-HFD mice. This phenotype was not explained by a simple reduction in cumulative caloric intake. Interestingly, the increased inflammatory markers mRNA expression (CD68, CCL2) observed in EAT of WT-HFD mice, was reduced in KO-HFD mice. Experiments are under investigations to further characterize mechanisms contributing to the resistance of mPGES-1 KO mice to diet-induced obesity. Altogether, these results suggest that inhibition of mPGES-1 may prevent diet-induced obesity in mice.

Table 1. mRNA expression of mPGES-1 in EAT of diet-induced obese mice.

\begin{tabular}{lcc}
\hline Measures & C57BL/6-NC $(\mathrm{n}=10)$ \\
\hline Final body weight gain (g) & $8 \cdot 07 \pm 0 \cdot 38$ \\
mPGES-1 mRNA expression - EAT & $1 \cdot 00 \pm 0 \cdot 20$ \\
\hline
\end{tabular}

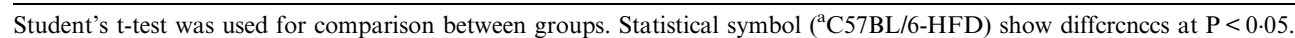

Table 2. Impact of mPGES-1 invalidation on diet-induced adiposity.

\begin{tabular}{|c|c|c|c|c|}
\hline Measures & VVT-NC $(n=7)$ & KO-NC $(n=6)$ & WT-HFD $(\mathrm{n}=8)$ & KO-HFD $(n=6)$ \\
\hline Final body weight gain $(\mathrm{g})$ & $3.61 \pm 0.64$ & $3.08 \pm 0.25$ & $7 \cdot 52 \pm 0.70^{a}$ & $4.34 \pm 0.63^{b c}$ \\
\hline Cumulative caloric intake (kcal) & $1120 \cdot 38 \pm 14 \cdot 34$ & $1022 \cdot 20 \pm 25 \cdot 94^{\mathrm{a}}$ & $1082 \cdot 11 \pm 27 \cdot 56$ & $1015.78 \pm 18.93^{a}$ \\
\hline Total adiposity ( $\%)$ & $10 \cdot 7210 \cdot 61$ & $10 \cdot 51 \pm 0 \cdot 91$ & $27 \cdot 34 \pm 2.74^{\mathrm{a}}$ & $17.99 \pm 1.76^{\circ}$ \\
\hline CCL2 mRNA expression - EAT & $1 \cdot 00 \pm 0.21$ & $1.01 \pm 0.33$ & $2.56 \pm 0.59^{\mathrm{a}}$ & $1.08 \pm 0.21^{\mathrm{c}}$ \\
\hline CD68 mRNA expression - EAT & $1.00 \pm 0.07$ & $1.07 \pm 0.21$ & $1.85 \pm 0.21^{\mathrm{a}}$ & $1 \cdot 29 \pm 0 \cdot 14^{\mathrm{c}}$ \\
\hline
\end{tabular}

One-way ANOVA was used for comparison groups, and Fisher's test for post-hoc analysis. Statistical symbols ( ${ }^{\mathrm{a}} \mathrm{WT}-\mathrm{NC}$, ${ }^{\mathrm{b}} \mathrm{KO}-\mathrm{NC}$, $\left.{ }^{\mathrm{c}} \mathrm{WT}-\mathrm{HFD}\right)$ show differences at $\mathrm{P}<0 \cdot 05$.

1. Thaler JP, Yi CX, Schur EA, et al. (2012) J Clin Invest 122, 153-162.

2. Strissel KJ, Stancheva Z, Miyoshi H et al. (2007) Diabetes 56, 2910-2918.

3. Richelsen B \& Pedersen SB (1987) Endocrinology 121, 1221-1226.

4. $\mathrm{Xu} \mathrm{H}, \mathrm{Fu}$ J-L, Miao Y-F et al. (2016) J Mol Cell Biol 8, 518-529.

5. Pecchi E, Dallaporta M, Jean A et al. (2009) Physiol Behav 97, 279-92. 